\title{
What Path Monitor: A Brief Note on Quantum Cognition and Quantum Interference, the Role of the Knowledge Factor
}

\author{
Elio Conte ${ }^{1,2}$ \\ ${ }^{1}$ School of Advanced International Studies on Applied Theoretical and Non-Linear Methodologies of Physics, \\ Bari, Italy \\ ${ }^{2}$ Department of Neuroscience and Sense Organs, University Aldo Moro, Bari, Italy \\ Email: elio.conte@fastwebnet.it
}

Received 1 February 2015; accepted 22 February 2015; published 26 February 2015

Copyright (c) 2015 by author and Scientific Research Publishing Inc.

This work is licensed under the Creative Commons Attribution International License (CC BY). http://creativecommons.org/licenses/by/4.0/

(c) ()

\begin{abstract}
We discuss a celebrated experiment of quantum mechanics to evidence that quantum mechanics delineates a novel feature of our reality in which cognition enters as primary element, strongly linked $a b$ initio to the dynamics of matter.
\end{abstract}

Keywords

Quantum Cognition, Human Cognition, Quantum Mechanics, Quantum Interference

\section{Introduction}

Cognition is the mental process of knowing, including aspects such as awareness, perception, reasoning, and judgment. It relates what comes to be known, perception, reasoning or intuition, and knowledge.

One of our long-range goals is to understand the neural underpinnings of human emotion and examine how cognitive and emotional brain systems interact in the generation of complex behaviour. Cognitive science is presently an advanced discipline but we are currently a long way from a thorough understanding about the cognitive mechanisms and the relationship between cognition and emotion.

Here we discuss a different approach to stimulate consideration and comments.

Our historical position in physical science as well as in psychology and neuroscience is to retain that matter is placed in space and time existing as an objective entity, independent from our human mental entities. They operate in the abstract, in a condition of total separation and independence, but also one of constant interaction 
with external objective material reality. However, in recent years some studies have started to delineate a possible, different perspective, indicating the possibility that actually the human cognitive dynamics arises $a b$ initio in strong relation with the material sphere and prospecting that the conceptual foundations of quantum mechanics could have an elective role in understanding the way in which primary elements of cognition on one hand and material reality are able to coexist just $a b$ initio. Generally speaking, the so-called quantum cognition has developed in recent years. In 1980-1986, the author of the present communication started with some initial contributions (see Conte, 2011c for details). A. Y. Khrennikov started to study the problem and Khrennikov and this author have other contributions, starting in 2003 until today. For a comprehensive review one may look at (Khrennikov, 2010) and (Conte, 2011c). In 1991, (Aerts, 1991) considered deeply the role of quantum mechanics obtaining results in quantum cognition as did others, such as P. D. Bruza and K. Kitto, starting in 2007, J. R. Busemeyer and E. M. Pathos in 2006 (see Busemeyer \& Bruza, 2012). In 1995-2003, D. M. Snyder also made important contributions (Snyder, 1995) as well as other authors. Now we may conclude that quantum cognition is viable.

We now have decisive experimental evidence regarding the existing quantum interference during perceptivecognitive performance in humans (Conte et al., 2007, 2009, 2011c, 2012). We performed experiments by using ambiguous figures but also the Stroop effect, testing cognitive anomalies as an example the conjunction fallacy (See Conte et al., 2011; Conte, 2011c, 2011d and references therein for a complete list of the papers).

In this paper the basic result we have reached is as follows.

As previously outlined, we are accustomed to consider reality as affected by a definite dualism. On one side, we have matter that exists per se independently from our observation and measurement. On the other side, we have human cognitive structure and function. It is able to receive inputs from the outside sphere and to elaborate such information by performing semantic acts and decisions.

The conclusion arising from our studies based on quantum mechanics, seems to move in an opposite direction. This is to say that, according to such results, quantum mechanics contains some basic features evidencing that its origin is in logic and cognition. It contains ab initio indication of our primary cognitive elements (Conte, 2011a, 2013a, 2013b). In particular, the wave function of quantum mechanics, that is the pillar of the theory, must be considered as a factor of knowledge that engages our cognitive performance.

The subsequent conclusion is that there are stages of our reality in which it is impossible to unconditionally define the truth. Logic, language, and thus cognition enter with so fundamental a role in quantum mechanics because there are levels of our reality in which the fundamental features of cognition and thus of logic and language, and thus the conceptual entities, acquire the same importance as the features of what is being described. At this level of reality, we cannot separate the features of matter per se from the features of cognition, logic, and the language that we use to describe it. Conceptual entities are inseparable from the object of cognitive performance.

This is a statement that seems to completely subvert our traditional way of approach to cognitive science and opens new and interesting perspectives in this field.

The aim of the present paper is to give a brief elaboration, entirely based on a quantum mechanical formulation and giving basic support to the previous thesis.

\section{The Basic Theoretical Elaboration}

Let us recall one basic postulate of the theory.

Any physical system is described by a state vector $|\psi\rangle$ which lives in a Hilbert space, a complex vector space equipped with an inner product $\langle\varphi \mid \psi\rangle$. A state vector has unit norm $\langle\psi \mid \psi\rangle=1$.

According to the Superposition Principle, any two state vectors (in a Hilbert space) of a quantum mechanical system, can be linearly added together to form another valid state of the system: For $|\psi\rangle,|\varphi\rangle \in H$ we have the state

$$
|\Psi\rangle=a|\psi\rangle+b|\varphi\rangle
$$

with $a, b \in C$ and $|a|^{2}$ probability for state $|\psi\rangle$ and $|b|^{2}$ probability for state $|\varphi\rangle$ and $|\psi\rangle$ and $|\varphi\rangle$ forming an orthonormal basis. 
This is the basic physical and mathematical elaboration that of course is followed with reservation from psychologists, since correctly they do not have direct competence on such disciplines. Of course we can reject the formulation strictly based on physics and mathematics and closely observe the arising and direct implication. We are faced here with some quantum principles that of course are applied in studies on the application of quantum mechanics in cognitive science. They are able to crush our more traditional approach to reality. We have recalled previously the Superposition Principle: it admits the possibility of existence of multiple states simultaneously. This is equivalent to considering that the reality outlined by quantum mechanics does not have a direct counterpart in the Boolean logic scheme where, for example, a traffic light is red or green. The Superposition Principle admits instead that red and green coexist simultaneously, at least to the level of ontological potentiality. In brief, the Superposition Principle responds to the And rather to the Or logic. While at the cognitive level it is not so difficult to accept that two mental states, corresponding to two logic alternatives, may coexist at a cognitive level, it remains rather difficult to accept, for example, that the spin z-projection of a particle (thus in this case considering not mental entities but matter) engages its dynamics using both its possible alternatives at the same time (spin up and down). However, the solid validity of the Superposition Principle is well established in the theory. The presence of the Superposition Principle causes the occurrence of many purely quantum mechanical effects, such as interference in the double slit experiment and entanglement of quantum systems.

There is still another feature that is of importance. When we consider states $|\varphi\rangle$ and $|\psi\rangle$, we admit that they are orthonormal and this seems to recover again a logic counterpart; that is to say either $|\varphi\rangle$ or $|\psi\rangle$. In other terms, in absence of observation and measurement, only the non-Boolean counterpart logic of the And, that is to say of coexisting alternatives, exists. If we consider a different knowledge factor, or a different knowledge condition, the dynamics of the quantum system is modified. During observation and measurement the traditional Boolean feature of the Or alternatives remains to operate.

Let us examine the problem of quantum interference.

There is in quantum physics a traditional experiment that represents a dilemma. The double-slit experiment is considered one of the most beautiful experiments in physics. It demonstrates simply and completely the strange features of quantum mechanics and of our quantum reality.

A device is spraying particles at a screen in which there are two opening slits which may or may not be covered. First, suppose the experiment is carried out with only one slit opened, say Slit 1 . The particles arrive on the detecting screen. We represent this experimental outcome by a curve $P_{1}(x)$ which is simply such that $P_{1}(x) \delta(x)=$ probability of a particle landing in the range $(x, x+\delta x)$. If we cover this slit and open the other, we will have producing a curve $P_{2}(x)$ similar to $P_{1}(x)$ according to the following picture (Figure 1 ).

This is of course exactly what we expect when performing such experiment at a macroscopic level. Finally, we admit that both slits are opened. We would then observe the particles would sometimes come through Slit 1 and sometimes through Slit 2, varying between the two possibilities in a random way, producing two piles behind each slit in a way that is simply the sum of the results that would be observed with one or the other slit opened.

Consequently we should have

$$
P_{12}(x)=P_{1}(x)+P_{2}(x)
$$

Instead, according to quantum mechanics we have

$$
P_{12}(x)=P_{1}(x)+P_{2}(x)+2 \sqrt{P_{1}(x) P_{2}(x)} \cos \vartheta \neq P_{1}(x)+P_{2}(x)
$$

We obtain what it is called a typical quantum interference pattern where a new term is given by

$$
2 \sqrt{P_{1}(x) P_{2}(x)} \cos \vartheta .
$$

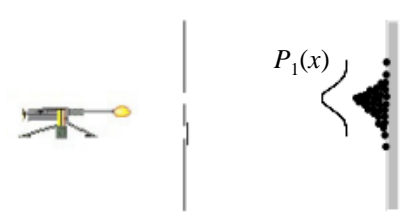

(a)

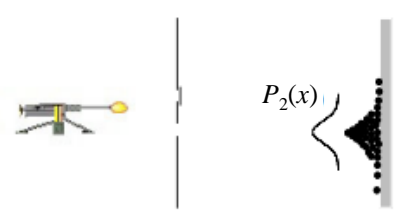

(b)

Figure 1. The experiment with slit 1 or slit 2 open. 
Let us explain how cognition enters in this experiment.

According to quantum mechanics and to the principle previously exposed, we have a superposition principle (simultaneous superposition of alternatives) and a wave function [that no more is a factor of knowledge]. Using such two statements, we describe the state of the particle just after the two slits:

$$
|\Psi\rangle=\frac{1}{\sqrt{2}}\left(\left|\psi_{1}\right\rangle+\left|\psi_{2}\right\rangle\right)
$$

where $\left|\psi_{1}\right\rangle$ denotes the state of the particle that has gone through Slit 1 , and $\left|\psi_{2}\right\rangle$ denotes the state of the particle through Slit 2.

This is our starting condition of knowledge factor. The $|\Psi\rangle$ represents our knowledge factor, our knowledge condition. [The rule here is that reality cannot be described if we renounce to characterize the factor of knowledge, i.e. the knowledge condition, then our cognitive position].

It is evident that here we have different cognitive conditions. The first is that we do not observe the system when the particle is travelling by Slit 1 or 2 . In this case we do not know anything about its pattern. This is the first case and our knowledge condition is represented by the function previously written, having the two coexisting alternatives.

The extraordinary feature of this experiment is that in the absence of any human performance about the path of the particle, say, in absence of what path monitor, the experiment gives as result the interference that appears in Figure 2, and is given by the term of quantum interference previously written. In substance, in absence of our observation, finalized to establish what slit the particle passed too, interference is present and we may represent it by the following Figure 2 .

This is the situation in which the probability for a particle hitting the screen violates $P_{12}(x)=P_{1}(x)+P_{2}(x)$ owing to the presence of the quantum interference term $2 \sqrt{P_{1}(x) P_{2}(x)} \cos \vartheta$.

Of course this is our first possible cognitive condition. It is that one that we do not know anything about the path taken by the particle.

Of course we have another cognitive condition. Roughly speaking, we may admit that we place an observer equipped with a bright flashlight (actually a proper device) near one of the two slits so that each time a particle starts we may have direct cognition of its path. In fact, suppose the flashlight is located at Slit 1, if particle travels by Slit 1 we see it and if it passes by Slit 2 at the same time, our cognitive apparatus elaborates that it passed by Slit 2 since we did not see it passing by Slit 1 . The same thing happens selecting to observe Slit 2 instead of 1 (Snyder, 1995).

Note that in this manner we, as human entities, perform only a pure cognitive act. In particular we do not disturb the particle when it passes by Slit 2 and we are located at the Slit 1 or viceversa. We only explain a role of cognition.
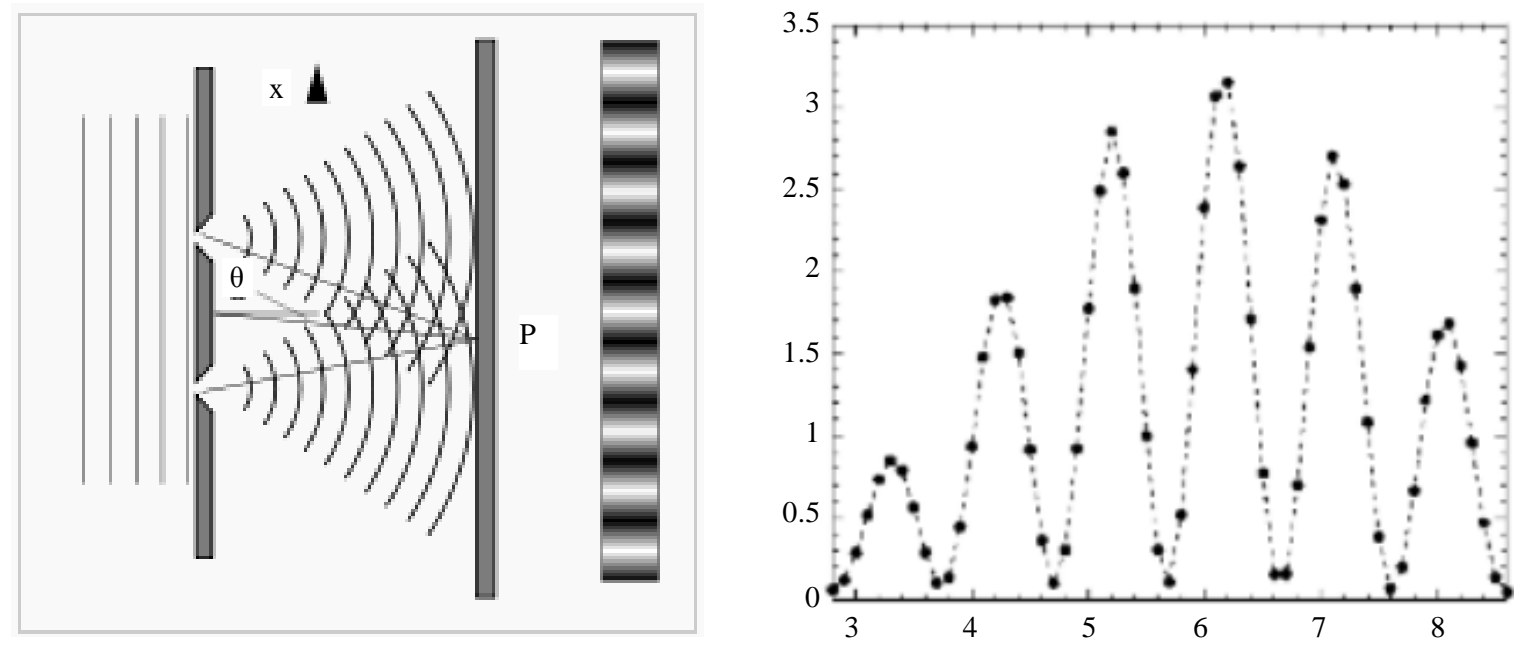

Figure 2. Interference pattern when both slits are open. 
However, the situation is now profoundly changed in respect to the first case. We have now introduced in our experiment a modified factor of knowledge, a what path monitor. This is the profound reason to attribute valuable importance to studies in quantum cognition and in particular to the principle that we advanced previously: there are stages of our reality in which we no more may conceive matter entities independently from the cognition that we have about it. According to the rules of quantum mechanics, if the factor of knowledge is changed, we have to modify the formal description according to the new situation. There are stages of our reality in which we no more may consider the old dualism and to separate the features of matter per se from the features of cognition, logic and of the language that we use to describe it. The knowledge factor always enters as the dominant factor in this experiment. In absence of any observation, we have a kind of wave function. Instead, if our aim is to attempt to identify what path the particle crosses, we need to introduce another and specific factor of knowledge. This is to say that we have to introduce a what path monitor to be able to perform a semantic act. Any semantic act finally relates cognition. In quantum mechanical terms, we have to represent the human cognitive mental state and we represent here it by $\left|e_{0}\right\rangle$.

Now, we will have a new wave function, a new knowledge function represented by $|\Psi\rangle\left|e_{0}\right\rangle$.

According to the rules of quantum mechanics, under a linear transformation, the state including a what path monitor will evolve giving

$$
|\Psi\rangle\left|e_{0}\right\rangle \rightarrow \frac{1}{\sqrt{2}}\left(\left|\psi_{1}\right\rangle\left|e_{1}\right\rangle+\left|\psi_{2}\right\rangle\left|e_{2}\right\rangle\right)
$$

Being $\left|e_{1}\right\rangle$ and $\left|e_{2}\right\rangle$ the two alternatives cognitive mental states coexisting at the level of our cognitive performance.

Let us perform only some brief mathematical developments. We introduce such physical elaboration here only for the reason of physical rigour that is due. But substantially we are calculating only the distribution of the location of the position of the particle when it reaches the screen. Calculate the reduced density operator when we are in presence of human cognitive performance represented, as previously said by the two cognitive alternative states, $\left|e_{1}\right\rangle$ relating when particle travels by Slit 1 and $\left|e_{2}\right\rangle$ when particles travels by Slit 2 . If we have the patience to observe the physical developments we observe that the reduced density operator

$$
\rho=\frac{1}{2}\left(\left|\psi_{1}\right\rangle\left\langle\psi_{1}|+| \psi_{2}\right\rangle\left\langle\psi_{2}|+| \psi_{1}\right\rangle\left\langle\psi_{2}\left|\left\langle e_{2} \mid e_{1}\right\rangle+\right| \psi_{2}\right\rangle\left\langle\psi_{1}\right|\left\langle e_{2} \mid e_{1}\right\rangle\right)
$$

immediately gives again the quantum interference term that experimentally gives the interference pattern that we have reproduced in Figure 2.

The particle density at position $z$ (we called it previously as $x$ ) will be given by

$$
\rho(z)=\langle z|\rho| z\rangle=\frac{1}{2}\left|\psi_{1}(z)\right|^{2}+\frac{1}{2}\left|\psi_{2}(z)\right|^{2}+\operatorname{Re}\left(\psi_{1}(z) \psi_{2}^{*}(z)\left\langle e_{2} \mid e_{1}\right\rangle\right)
$$

The term

is the interference term.

If we have not quantum interference and this is to say that the particle goes through Slit 1 or 2 , the previous particle density at position $z$ becomes

$$
\rho(z)=\frac{1}{2}\left|\psi_{1}(z)\right|^{2}+\frac{1}{2}\left|\psi_{2}(z)\right|^{2}=P_{1}+P_{2}
$$

as previously explained.

We may observe that this is really the case in this new situation in which the cognitive condition, the what path monitor, is acting. In fact we observe that the interference term contains the factor

$$
\alpha_{S}=\left\langle e_{2} \mid e_{1}\right\rangle
$$

that must be considered, and it involves our cognitive mental entities that must be alternative and thus responding to a Boolean yes/no, Slit 1 or Slit 2 logic.

This cognitive condition crucially requires that the factor $\alpha_{S}=\left\langle e_{2} \mid e_{1}\right\rangle$ is zero. If the factor $\alpha_{S}=\left\langle e_{2} \mid e_{1}\right\rangle$ is zero, the interference term disappears and we return to the classical case with particles going through Slit 1 or 2 
with given probabilities. However, in order to realize such a condition, a what path monitor must be included in the experiment. This is to say that a semantic act must be included by the cognitive entity represented by $\left|e_{0}\right\rangle$ and linearly transformed according to the (1.1). This is actually what happens at the experimental level.

\section{Conclusion}

In conclusion, only a cognitive entity, able to perform a condition of knowledge (a what path monitor), realizes a situation that is totally different from the case in which the particle goes across the slits in total absence of our awareness. Our cognition determines Boolean alternatives that in our case are characterized from $\left\langle e_{2} \mid e_{1}\right\rangle=0$. The presence of a knowledge factor is always determinant in such experiment as well as we have discussed in detail also in other equivalent experimental conditions. Conceptual entities non more may be separated from the object of cognitive performance. Of course, consciousness by itself is the abstract entity obeying to the rules of quantum mechanics (Conte, 2014, 2015). From a psychological point of view there is finally a result that seems to close the circle of the present discussion. As shown in detail by us in previous papers (Conte, 2011a, 2011b), we obtain the same kind of interference, here discussed by using particles (and thus matter) in the well known two slits experiment of quantum physics, using instead logic statements.

\section{References}

Aerts, D. (1991). A Mechanistic Classical Laboratory Situation Violating the Bell Inequalities with 2sqrt(2), Exactly in the Same Way as Its Violation by the EPR. Helvetica Physica Acta, 64, 1-23.

Busemeyer, J. R. \& Bruza, P. D. (2012). Quantum Models of Cognition and Decisions. Cambridge: Cambridge University Press. http://dx.doi.org/10.1017/CBO9780511997716

Conte, E. (2011a). On the Logical Origins of Quantum Mechanics Demonstrated by Using Clifford Algebra: A Proof That Quantum Interference Arises in a Clifford Algebraic Formulation of Quantum Mechanics. Electronic Journal of Theoretical Physics, 8, 109-126.

Conte, E. (2011b). On the Logical Origins of Quantum Mechanics Demonstrated by Using Clifford Algebra. Neuroquantology, 9, 231-242. http://dx.doi.org/10.14704/nq.2011.9.2.397

Conte, E. (2011c). Advances in Application of Quantum Mechanics in Neuroscience and Psychology: A Clifford Algebraic Approach. New York: Nova Science Publishers.

Conte, E. (2013a). Are Information, Cognition, and the Principle of Existence Intrinsically Structured in the Quantum Model of Reality? Advanced Studies in Theoretical Physics, 7, 797-818.

Conte, E. (2013b). A Clifford Algebraic Analysis Gives Mathematical Explanation of Quantization of Quantum Theory and Delineates a Model of Quantum Reality in Which Information, Primitive Cognition Entities and a Principle of Existence Are Intrinsically Represented Ab Initio. World Journal of Neuroscience, 3, 157-170. http://dx.doi.org/10.4236/wjns.2013.33021

Conte, E. (2014). Answer to Giancarlo Ghirardi: Quantum Superpositions and Definite Perceptions: Envisaging New Feasible Experimental Tests. A Novel Proposal for Quantum Mechanics, Perception and Cognitive Science? International Journal of Theoretical Physics. http://dx.doi.org/10.1007/s10773-014-2259-6

Conte, E. (2015). Additional Comments Added to Our Recent Answer to G. Ghirardi. Journal of Modern Physics, 6, 12-15. http://dx.doi.org/10.4236/jmp.2015.61002

Conte, E., Khrennikov, A. Y., Todarello, O., De Robertis, R., Federici, A., \& Zbilut, J. P. (2011). On the Possibility That We Think in a Quantum Mechanical Manner: An Experimental Verification of Existing Quantum Interference Effects in Cognitive Anomaly of Conjunction Fallacy. Chaos and Complexity Letters, 4, 123-136.

Conte, E., Khrennikov, A. Y., Todarello, O., Federici, A., Mendolicchio, L., \& Zbilut, J. P. (2009). Mental States Follow Quantum Mechanics during Perception and Cognition of Ambiguous Figures. Journal of Open Systems and Information Dynamics, 16, 1-17.

Conte, E., Santacroce, N., Laterza, V., Conte, S., Federici, A., \& Todarello, O. (2012). The Brain Knows More Than It Admits: A Quantum Model and Its Experimental Confirmation. Electronic Journal of Theoretical Physics, 9, 72-110.

Conte, E., Todarello, O., Federici, A., Vitiello, F., Lopane, M., Khrennikov, A. Y., \& Zbilut, J. P. (2007). Found Experimental Evidence of Quantum Like Behavior of Cognitive Entities. An Abstract Quantum Mechanical Formalism to Describe Cognitive Entities and Its Dynamics. Chaos, Solitons and Fractals, 3, 1076-1088.

http://dx.doi.org/10.1016/j.chaos.2005.09.061

Khrennikov, A. (2010). Ubiquitous Quantum Mechanics. Berlin: Springer-Verlag Berlin Heidelberg.

Snyder, M. D. (1995). On the Quantum Mechanical Wave Function as a Link between Cognition and the Physical World: A Role for Psychology. http://cogprints.org/2196/ 
Scientific Research Publishing (SCIRP) is one of the largest Open Access journal publishers. It is currently publishing more than 200 open access, online, peer-reviewed journals covering a wide range of academic disciplines. SCIRP serves the worldwide academic communities and contributes to the progress and application of science with its publication.

Other selected journals from SCIRP are listed as below. Submit your manuscript to us via either submit@scirp.org or Online Submission Portal.
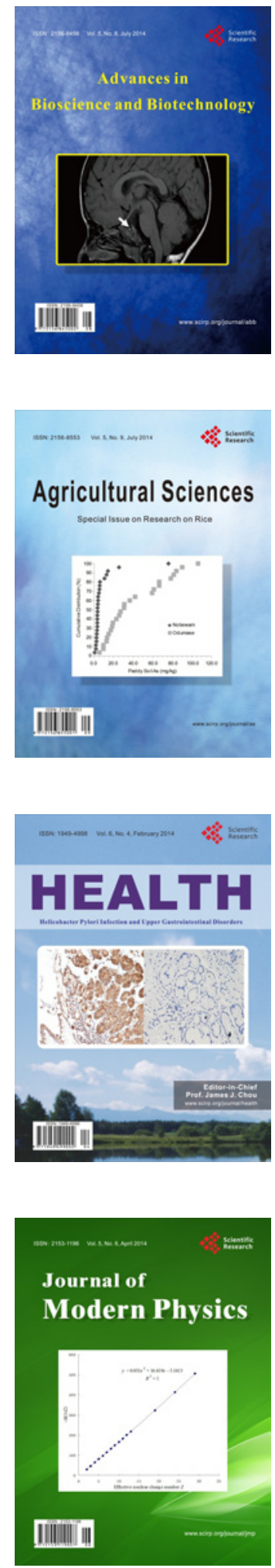
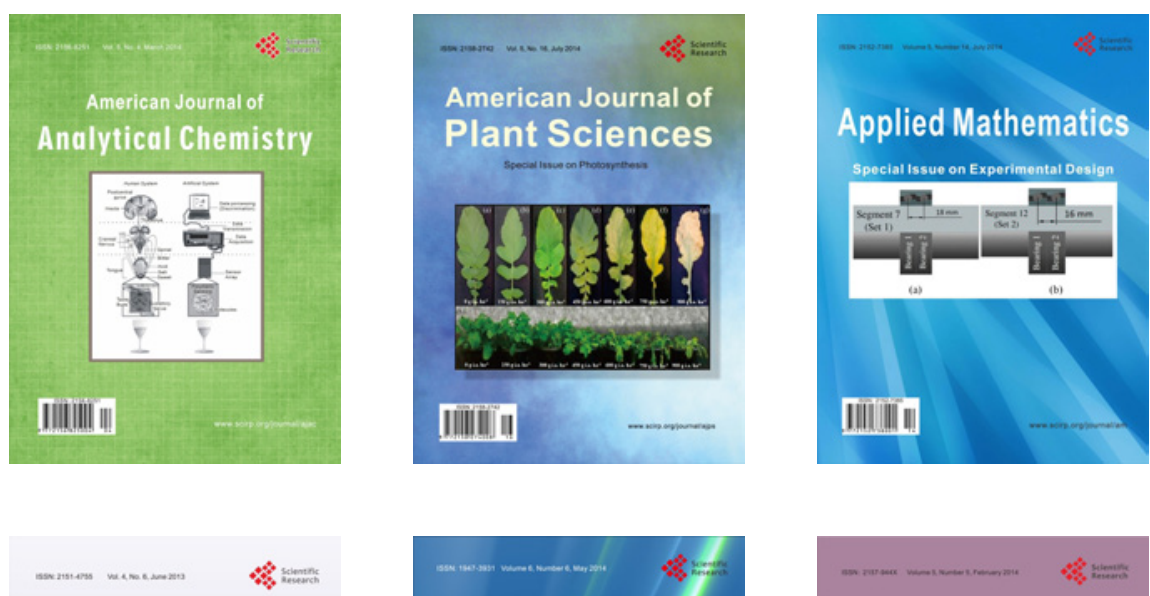

Creative Education
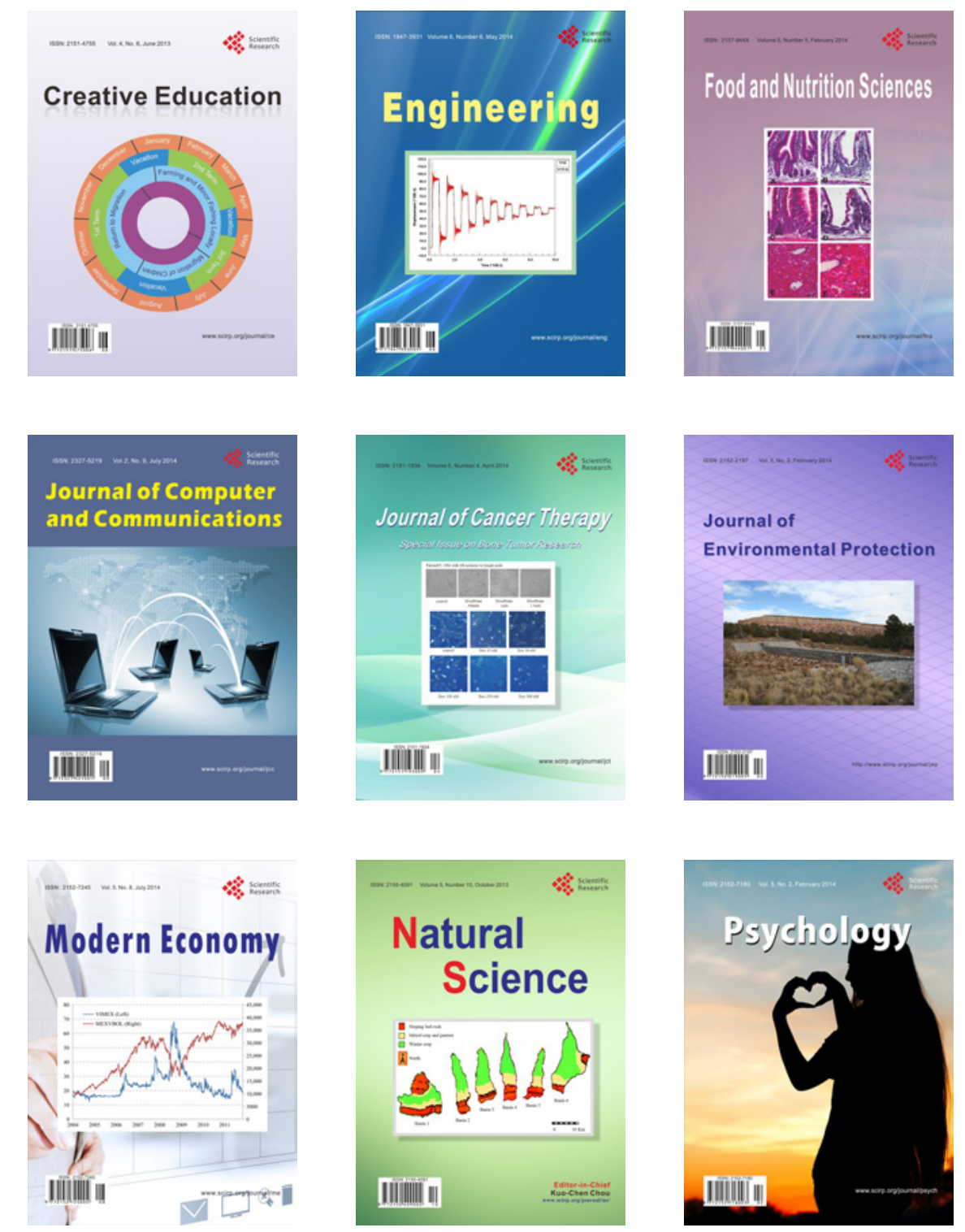